\title{
Multicultural Competence: The Cinderella of Psychology
}

\author{
La Keita Carter* \\ Psychology Division, Loyola University Maryland, United States
}

\author{
Received: November 16, 2013; Accepted: December 11, 2013; Published: December 13, 2013 \\ *Corresponding author: La Keita Carter, Psychology Division, Loyola University Maryland, United States, Tel: 410- 617-1200; E-mail: \\ Ldcarter@loyola.edu
}

"I never considered myself privileged just because I was a male." "Of course, I am multi-culturally competent; I am bilingual." "Diagnosing is standardized; culture should have nothing to do with it."

Sadly, the authors of these comments aren't those who are still in training to be psychologists. It's the psychology professionals who are the proud owners of these comments. Clinical supervisors, training directors and department chairs have fronted these comments, which, at the least, stem from ignorance and, at best, demonstrate multicultural incompetence, intolerance and insensitivity.

Ignorance is the state of being unaware, uninformed or unfamiliar. Let's face it; we are all ignorant to something. For example, most psychologists are totally unaware of the ethical principles that guide other professions like medicine or law. There's nothing wrong with ignorance in some situations, and sometimes ignorance is unavoidable. However, are there times when ignorance is unacceptable?

We psychologists have come to use ignorance as our insanity plea. If you can't explain why you made a specific decision after considering the whole client, cultural context and all, you can register a plea of ignorance like a defense attorney does on behalf of a client when she can't find any logical motive for her client's law-breaking behavior. There may be times when the insanity plea appropriately fits, just like there are times when ignorance is acceptable. Some might ask, "When can you appropriately claim ignorance in the multicultural competence arena?" However, the question should be, "Why should we not be using ignorance as an excuse for multicultural incompetence?"

Ethics and multicultural competence are sisters in the practice of psychology. They were birthed from the same psychological principles of beneficence (Principle A) and respect (Principle E). In other words, the American Psychological Association implemented and currently enforces the requirement that all psychologists have to obtain continuing education in ethics and multicultural competence because of the overriding principles of doing good to our clients and respecting their cultural differences. Therefore, the first reason that the 'ignorance' flag won't fly with multicultural competence is because it doesn't fly when it comes to its equally-important sister, ethics. APA as well as state psychology boards have made it abundantly clear that not knowing an ethical standard is not an acceptable excuse for violating even one of them. Yet, we accept the excuse for its sister. We are warned about knowing "The Code" so that we can keep active licenses. Are we getting the same messages of caution when it comes to multicultural competence?

It seems that APA is even confused on this issue. While we are required to incorporate this specific training into our classrooms, therapy rooms and assessment rooms, it isn't even listed on the front page of apa.org's "Psychology Topics" section. Further investigation reveals that the only way to generate magazine articles, newsletters, reports and the like on apa.org is to type multicultural competence into the search box at the top of the website. It almost seems like this area of psychology is the stepsister of ethics, not the full-blooded sister who gets psychologists' equal amount of professional attention, commitment and concern.

Another problem with a psychologist waving this alltoo-often-used ignorance flag is that licensed psychologists shouldn't be ignorant in this area. They should have more than basic knowledge of what it means to practice psychology from a multicultural standpoint. Don't we renew our licenses every two years and demonstrate that we have done the bare minimum of maintaining our multicultural competence by acquiring at least three hours of continuing education in this area? Psychologists should not make the effort to be multiculturally competent; they should be multiculturally competent Period.

It seems that the theory of all psychologists being competent in this area is more important that they practice of it. If we really wanted to be multiculturally competent clinical supervisors, we would train students to carefully consider their clients in a cultural context, and we could start by teaching them the difference between race, ethnicity and culture. Unfortunately, most psychologists don't know those differences; therefore, they can't pass that knowledge onto their students who are eager to be molded.

If we really wanted to be multiculturally competent professors, we wouldn't stick a journal article on multiculturalism at the end of the semester to meet some departmental requirement. It would be infused with all clinical training. That is, part of the discussion in tests and measurement's classes should be how 
different cultures historically respond to standardized tests. Theories of counseling classes could incorporate empirical data about how different groups of people approach, progress and terminate from treatment.

If we really wanted to be multiculturally competent psychologists, we would treat multicultural competence like we treat other aspects of psychology in which we want to be proficient. If you had a number of clients who were interested in smoking cessation and you wanted to provide hypnosis, you would seek out more than one class per licensing period to get trained. You would attend as much training as you needed to obtain competence and then attend CE trainings to maintain that competence throughout the year.

If we really wanted to be multiculturally competent psychologists, we would pursue a journey of self-reflection to uncover our own biases, identity statuses and assumptions. We wouldn't just assume that, because we are fluent in two languages, we understand all clients from a cultural context. We wouldn't assume that the exposure that we got to multiculturalism in graduate school 12 years ago still applies to our field today or the people who have come to rely on us for mental health help.

A multiculturally competent psychologist would know that even the title of this piece is biased toward American women as other nationalities and even American men may not know the story of Cinderella and how she was a young girl who was treated poorly by her stepsisters just because she was different.

If we really wanted to be multiculturally competent psychologists, we would be multiculturally competent psychologists. 